\title{
Gut-microbiome Taxonomic Profiling as Non-invasive Biomarkers for the Early Detection of Alcoholic Hepatocellular Carcinoma
}

\author{
Jun Seok ${ }^{1,2}$, Ki Tae Suk ${ }^{1,2}$ \\ ${ }^{1}$ Institute for Liver and Digestive Diseases, ${ }^{2}$ Department of Internal Medicine, Hallym University College of Medicine, Chuncheon, Korea
}

Received Nov. 24, 2019

Revised Nov. 26, 2019

Accepted Nov. 26, 2019
Background/Aims: Hepatocellular carcinoma (HCC) is a prevalent form of primary liver cancer and the fifth leading cause of worldwide cancer mortality. Though early diagnosis of HCC is important, so far lack of effective biomarkers for early diagnosis of HCC has been a problem. In this study, we searched for potential functional biomarkers of alcoholic HCC by using metagenomics approach.

Methods: Between September 2017 and April 2019, normal control $(n=44)$, alcoholic liver cirrhosis $(n=44)$, and alcoholic HCC $(n=13)$ groups were prospectively enrolled and analyzed. Gut microbiota was analyzed using the 16S-based microbiome taxonomic profiling platform of EzBioCloud Apps and analyzing system.

Results: There was a statistically significant difference among groups in diversity $(P<0.05)$. In the comparison of phylum between cirrhosis and HCC, Proteobacteria were increased and Bacteroidetes were decreased. Firmicutes were not significantly different among the three groups. In the taxonomic profiling, relative abundance of Lactobacillus in the cirrhosis and HCC groups showed richness $(P<0.05)$. In the biomarker analysis between cirrhosis and HCC, obiquinome Fe-S protein 3, global nitrogen regulator, Vesicle-associated membrane protein 7 , toxin YoeB, peroxisome-assembly ATPase, and nitrogen oxide reductase regulator were differently expressed $(P<0.001)$.

Conclusions: Alcoholic HCC showed different expressions in the stool taxonomy and biomarker compared with that of cirrhosis and control. Therefore, new biomarkers using stool analysis for alcoholic HCC are necessary. (J Liver Cancer 2020;20:32-40)

Keywords: Hepatocellular carcinoma; Biomarker; Taxonomy; Microbiome

\section{INTRODUCTION}

Liver cirrhosis and hepatocellular carcinoma (HCC) constitute the most chronic forms of liver disease and are designated as end-stage liver diseases. HCC, which has a 9.1\%

\footnotetext{
Corresponding author: Ki Tae Suk

Department of Internal Medicine, Hallym University College of Medicine, 77 Sakju-ro, Chuncheon 24253, Korea

Tel. +82-33-240-5826, Fax. +82-33-241-8064

E-mail; ktsuk@hallym.ac.kr

https://orcid.org/0000-0002-9206-9245
}

mortality rate worldwide, is the fifth most common cancer, and is considered a serious health burden worldwide. ${ }^{1}$

Current perspectives on the disease and promising treatments have been transformed by recent advances in basic and applied knowledge of the gut microbiota in humans. ${ }^{2}$ Human gut microbiota is set up in complex ecosystems containing a wide range of bacterial species, the total mass of which is approximately $1-2 \mathrm{~kg} .{ }^{3,4}$ In addition, bacterial species in the gut are closely related to the host, and play an important role in bacterial translocation, mucosal immune sys- 
tem, and vitamin production. ${ }^{5}$ Since the intestine is directly connected to the liver, gut microbiota disorders are causally related to liver diseases. The portal vein mediates the movement of nutrients and microbiota-related components along the enterohepatic axis. ${ }^{6-9}$

Recently, using a newly developed diagnostic method, a dramatic relationship between gut microbiome and liver cirrhosis, and gut microbiome and HCC has been observed. ${ }^{10}$ The intestinal environment consists of numerous bacteria in addition to archaea, eukaryotes, and viruses, all of which play an essential role in producing active metabolites, and maintaining homeostasis and important functions of a healthy host. Given its anatomical location, the liver is very closely related to the intestine. The liver is the first organ to be exposed to virulence factors derived from the intestine, including bacteria, damage-associated metabolites, and bacterial products, because it receives most of the blood and nutrient supply from the intestine through the portal vein. ${ }^{11,12}$ Changes in the gut microbial community promote increased translocation of event bacteria and active metabolites, known as endotoxemia, leading to barrier disruption and triggering systemic inflammation. However, this has a severe impact on the progression of chronic liver damage such as nonalcoholic fatty liver disease and alcoholic liver disease. ${ }^{13,14}$ These complications are often preterminal events in cirrhosis, and prevention and early management can improve the prognosis and progression to HCC. Therefore, the aim of the present study is to compare the gut microbiome found in patients among the normal, alcoholic liver cirrhosis, and HCC groups, and find an effective functional biomarker for the early diagnosis of HCC.

\section{METHODS}

\section{Patients}

The prospective cohort study was carried out between September 2017 and April 2019. A total of 101 patients comprising normal controls $(n=44)$, alcoholic liver cirrhosis $(n=44)$, and alcoholic HCC $(n=13)$ groups were prospectively enrolled and analyzed. This prospective cohort study involved patients with cirrhosis and HCC who were followed-up at the Liver Unit of the Hallym University Medical Center. The diseases of the patients were treated regardless of the study.

Patients who were $>20$ years old, had liver function test with an aspartate aminotransferase (AST)/alanine aminotransferase (ALT) $>1$ and elevated AST (ALT) level, and an alcohol consumption history of more than $40 \mathrm{~g} /$ day (for women) and $60 \mathrm{~g} /$ day (for men) during the last 7 days before screening were enrolled. Their last drinks were within 48 hours prior to admission. The exclusion criteria were as follows: patients with viral hepatitis, nonalcoholic hepatitis, autoimmune hepatitis, pancreatitis, hemochromatosis, Wilson's disease, and drug-induced liver injury. This study was conducted in conformance with the ethical guidelines from the 1975 Helsinki Declaration as it is reflected by a prior approval by the institutional review board for human research in hospital participating in the trial (2016-134). Informed consent for enrollment was obtained from each participant.

Baseline evaluation was performed on complete blood count, liver function testing, and viral markers, and patients with alcoholic liver cirrhosis and alcoholic HCC were scanned at the abdomen (abdominal computed tomography). AST, ALT, total bilirubin, gamma-glutamyl transferase, albumin, prothrombin time, international normalized ratio, creatinine, and $\alpha$-fetoprotein were included for serum biochemical parameters. Tests for hepatitis viruses and the human immunodeficiency virus were conducted in all patients. Hepatitis A immunoglobulin G ( $\operatorname{IgG})$ and $\operatorname{IgM}$ antibodies were used for the detection of hepatitis A virus; the IgM antibody against the hepatitis B core antigen and the hepatitis B surface antibody against the hepatitis B surface antigen were used for the detection of hepatitis B virus; anti-hepatitis E IgG and $\operatorname{IgM}$ antibodies were used for the detection of hepatitis E virus; and anti-hepatitis $C$ antibodies with or without the presence of hepatitis C RNA were used for the detection of hepatitis $\mathrm{C}$.

Enrolled patients and control groups were subjected to stool sampling and clinical analysis. Clinical data was matched with the metagenomics data simultaneously. Fecal samples were obtained noninvasively in a plastic collection kit at any time during the day. All samples were stored at 
$-80^{\circ} \mathrm{C}$. In the case of cirrhosis and HCC, stool samples were collected upon admission at the hospital and kept in a refrigerator at $-80^{\circ} \mathrm{C}$. In the case of normal control, the subjects collected the stool samples at home, and kept stool box at $-20^{\circ} \mathrm{C}$ in a refrigerator. They sent stool box with the stools in iced state to the hospital, where the stool samples were received and kept at $-80^{\circ} \mathrm{C}$ in a refrigerator.

\section{Stool analysis for the metagenomics}

Metagenomic DNA was extracted with a QIAamp stool kit and amplification of the V3-V4 region of the bacterial $16 \mathrm{~S}$ rRNA gene was conducted using barcoded universal primers. Polymerase chain reaction (PCR) was performed with the following conditions: an initial denaturation at $95^{\circ} \mathrm{C}$ for 5 minutes, 20 cycles of $95^{\circ} \mathrm{C}$ for 30 seconds, $55^{\circ} \mathrm{C}$ for 30 seconds, and $72^{\circ} \mathrm{C}$ for 30 seconds, followed by a final extension at $72^{\circ} \mathrm{C}$ for 10 minutes. Purification of the amplicons was conducted with an Agencourt AMPure XP system (Beckman Coulter Inc., Sykesville, MD, USA), and quantification of the purified amplicons was conducted using PicoGreen and quantitative PCR. After pooling of the barcoded amplicons, sequencing was carried out using a MiSeq sequencer on the Illumina platform (ChunLab Inc., Korea) according to the manufacturer's specification.

Microbiome profiling was conducted with the 16S-based Microbial taxonomic profiling (MTP) platform of EzBioCloud Apps (ChunLab Inc., Seoul, Korea). After taxonomic profiling of each sample, the comparative MTP analyzer of EzBioCloud Apps was used for comparative analysis of the samples. In the MTP platform of ChunLab, preprocessing of the sequencing reads was conducted using the following five steps: 1) filtering of low-quality reads, 2) merging of the paired-end reads, 3) removal of barcode and primer sequences, 4) taxonomic assignment of the reads, and 5) removal of chimeric sequences. Taxonomic assignment of the reads was conducted with ChunLab's $16 \mathrm{~S}$ rRNA database (DB ver. PKSSU4.0). ${ }^{15}$ OUT picking was conducted with UCLUST and CDHIT with $97 \%$ of similarity cutoff. ${ }^{16}$ Then, Good's coverage, rarefaction, and alpha-diversity indices including Abundance-based Coverage Estimator (ACE), and the number of OTUs found in MTP were calculated. Betadiversity, including PCoA clustering, was shown in the comparative MTP analyzer. All $16 \mathrm{~S}$ rRNA sequences were deposited in the ChunLab's EzBioCloud Microbiome Database and the sequencing reads of the $16 \mathrm{~S}$ rRNA gene from this study were deposited in the NCBI Short Read Archive.

\section{Quantification of bacterial amounts by quanti- tative real-time PCR}

The relative amounts of total bacteria were measured using quantitative real-time PCR based on the $16 \mathrm{~S}$ rRNA gene. The $16 \mathrm{~S}$ rRNA gene was amplified using the primers 340F (5'-TCCTACGGGAGGCAGCAG-3') and 518R (5'-ATTACCGCGGCTGCTGG-3') with the TaKaRa PCR Thermal Cycler Dice Real Time System III (Takara Bio Inc., Kusatsu, Japan). Triplicate reactions were performed for each sample with a final volume of $25 \mu \mathrm{L}$ comprising $12.5 \mu \mathrm{L}$ of $2 \mathrm{X}$ SYBR Premix Ex Taq (Takara Bio Inc.), $10 \mu \mathrm{M}$ of each primer, and $2 \mu \mathrm{L}$ of DNA template (ten-fold diluted metagenomic DNA) or distilled water (negative control). The conditions for the reaction were as follows: initial denaturation at $95^{\circ} \mathrm{C}$ for 30 seconds; 40 cycles of denaturation at $95^{\circ} \mathrm{C}$ for 5 seconds, annealing at $60^{\circ} \mathrm{C}$ for 30 seconds. Standard curves were generated from parallel PCRs with serial log-concentrations $(1 \times$ $\left.10^{2}-1 \times 10^{8}\right)$ of the copy number of the $16 \mathrm{~S}$ rRNA gene from Escherichia coli w3110.

\section{Sequence analysis}

Raw sequence reads were merged, and sequences with short read lengths ( $<430 \mathrm{bp}$ of merged reads) or low quality score and chimeric reads were trimmed using USEARCH (ver. 11.0.667). Primer sequences were removed from the merged sequences. Then, the resultant sequences were stepwise clustered into operational taxonomic units (OTUs) by 97\% identity with the EzTaxon-e database, and taxonomic positions of representative sequences in each OTU cluster were assigned. ${ }^{17}$ To compare diversity indices among samples, the read numbers were normalized using random subsampling, and the diversity indices were calculated using 
Mothur. ${ }^{18}$ Principal coordinate analysis (PCoA) plots were generated to compare microbiota composition among samples. ${ }^{19}$ The significantly differential taxa between groups were determined using Linear Discriminant Analysis Effect Size. ${ }^{20}$

\section{Data analysis}

Unless otherwise stated, all clinical data are expressed as mean \pm standard deviation. A paired $t$-test, independentsamples $t$-test, and analysis of covariance were used to assess the significance of the data. The differences in microbiota between groups were analyzed using the Mann-Whitney $U$ test and Kruskal-Wallis test in the R software. Permutation tests were used to determine the significance of the PCoA plot result. Values of $P<0.05$ were considered as statistically significant. Routine blood test data were analyzed using statistical software (SPSS, version 19.0, SPSS, Inc., Chicago, IL, USA) and GraphPad Prism version 6.0 for Windows (GraphPad Software Inc., San Diego, CA, USA).

\section{RESULTS}

\section{Baseline characteristics of patients}

The mean age of the patients was $58.0 \pm 10.0$ years and 68 patients $(73.7 \%)$ were male. The number of the normal control group was $44(43.6 \%)$ and $20(47.7 \%)$ were male. The number of the alcoholic liver cirrhosis group was 44 (43.6\%) and $35(79.5 \%)$ patients were male. The number of the alcoholic HCC group patients was 13 (12.9\%) and 12 (92.3\%) patients were male (Table 1).

\section{Diversity of taxonomy}

We compared alpha diversity in the normal control, alcoholic liver cirrhosis, and alcoholic HCC groups using the Wilcoxon rank-sum test. There was a statistically significant difference between the normal control and the alcoholic liver cirrhosis groups $(P<0.001)$. In addition, the alcoholic HCC group showed statistically significant difference compared with the normal control group $(P<0.05)$. There was no statistically significant difference between the alcoholic liver cirrhosis and alcoholic HCC groups ( $P=0.177$ ) (Fig. 1).

Additionally, we compared beta diversity in the normal control, alcoholic liver cirrhosis, and alcoholic HCC groups. The alcoholic liver cirrhosis and alcoholic HCC groups had similar beta diversity, but the normal control group showed different map pattern (Fig. 2).

Table 1. Baseline characteristics of patients

\begin{tabular}{|c|c|c|c|c|}
\hline Variable & Normal control & Alcoholic LC & Alcoholic HCC & $P$-value ${ }^{*}$ \\
\hline Patient & $44(43.6)$ & 44 (43.6) & $13(12.9)$ & NS \\
\hline Age (years) & $62.5 \pm 8.7$ & $54.1 \pm 9.6$ & $55.4 \pm 9.6$ & NS \\
\hline Sex (male) & $21(47.7)$ & $35(79.5)$ & $12(92.3)$ & NS \\
\hline AST (IU/L) & $22.9 \pm 4.7$ & $104.8 \pm 151.4$ & $69.7 \pm 62.5$ & NS \\
\hline ALT (IU/L) & $19.0 \pm 7.6$ & $41.6 \pm 51.8$ & $38.1 \pm 46.3$ & NS \\
\hline$\gamma-G T(I U / L)$ & $26.1 \pm 18.5$ & $477.2 \pm 884.1$ & $355.8 \pm 321.0$ & NS \\
\hline Creatinine (mg/dL) & $0.9 \pm 0.2$ & $0.8 \pm 0.3$ & $0.8 \pm 0.2$ & NS \\
\hline AFP (ng/mL) & & $7.2 \pm 6.3$ & $318.3 \pm 746.8$ & NS \\
\hline Child-Pugh score & - & $6.2 \pm 2.3$ & $7.8 \pm 3.4$ & NS \\
\hline MELD score & - & $11.0 \pm 6.8$ & $11.1 \pm 6.5$ & NS \\
\hline
\end{tabular}

Values are presented as mean \pm standard deviation or number (\%).

LC, liver cirrhosis; HCC, hepatocellular carcinoma; NS, not significant; AST, aspartate aminotransferase; ALT, alanine aminotransferase; $\gamma$-GT, gamma glutamyl transferase; AFP, a-fetoprotein; MELD, model for end-stage liver disease.

*Independent $t$-test. 


\section{Stool analysis for metagenomics}

For comparison of the taxonomic composition at the phylum level, Proteobacteria increased in the alcoholic liver cirrhosis and alcoholic HCC groups compared with that in the normal control group. Composition of Firmicutes was not significantly different among the normal control, alcoholic liver cirrhosis, and alcoholic HCC groups. Verrucomicrobia appeared only in the cirrhosis group, whereas Actinobacteria appeared in both alcoholic liver cirrhosis and alcoholic HCC groups (Fig. 3).

We compared Lactobacillus taxonomic relative abundance in the normal control, alcoholic liver cirrhosis, and alcoholic HCC groups. There was a statistically significant difference
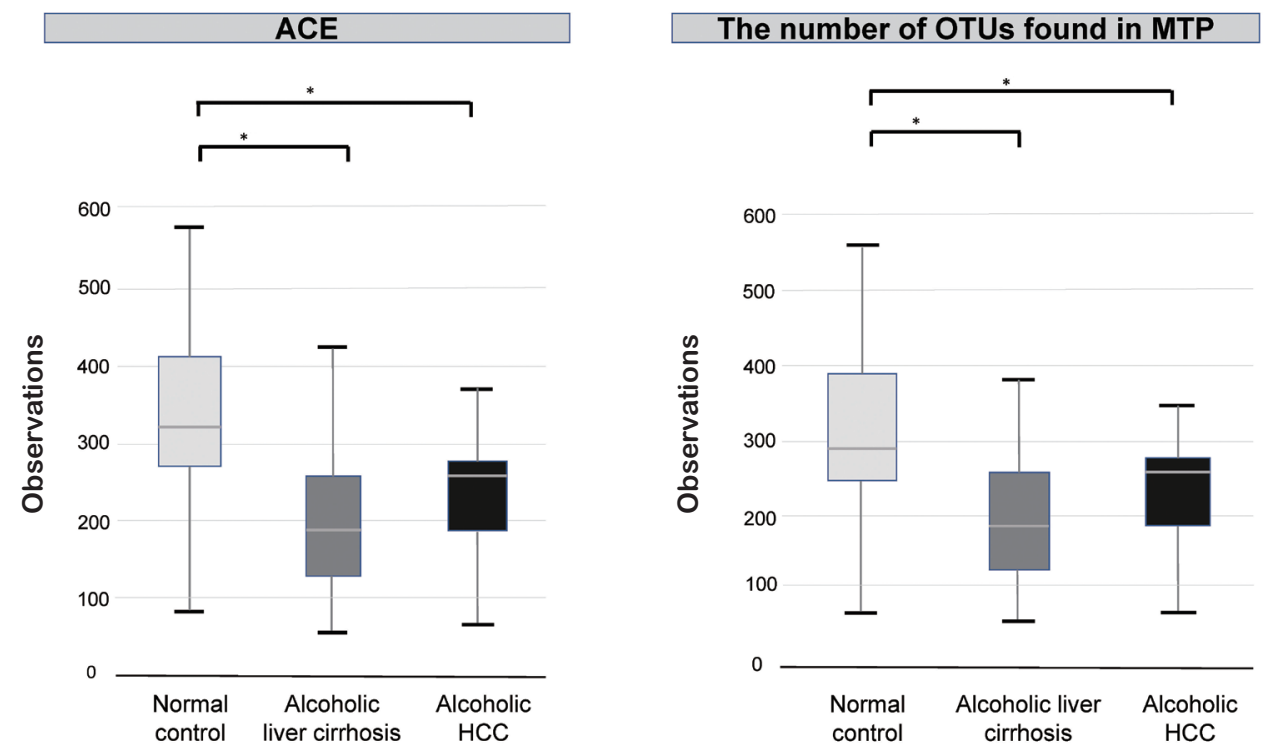

Figure 1. Alpha diversity in the normal control, alcoholic liver cirrhosis, and alcoholic hepatocellular carcinoma (HCC) groups. Alcoholic liver cirrhosis and alcoholic HCC groups showed decrease alpha diversity compared to that of the normal control group. ACE, Abundance-based Coverage Estimator; OTU, operational taxonomic unit; MTP, microbial taxonomic profiling. ${ }^{*} P<0.001$.

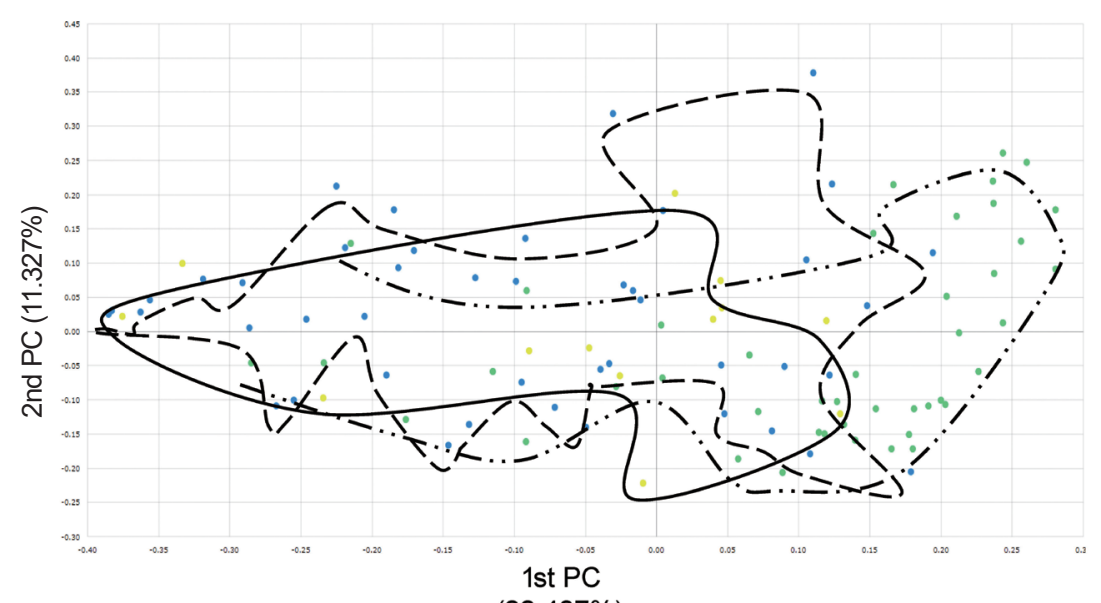

$(22.487 \%)$

Normal control Alcoholic liver cirrhosis Alcoholic HCC

Figure 2. Beta diversity in the normal control, alcoholic liver cirrhosis, and alcoholic hepatocellular carcinoma (HCC) groups. Alcoholic liver cirrhosis and alcoholic HCC groups had similar beta diversity and showed different patterns compared with that of the normal control group. 
between the normal control and the alcoholic liver cirrhosis groups $(P<0.001)$, and a statistically significant difference between the normal control and alcoholic HCC groups $(P<0.05)$. There was no statistically significant difference between the alcoholic liver cirrhosis and alcoholic HCC groups $(P=0.171)$ (Fig. 4).

Firmicutes to Bacteroidetes ratio was compared in the normal control, alcoholic liver cirrhosis, and alcoholic HCC groups. There were no statistically significant differences in all three groups (normal control group vs. alcoholic liver cirrhosis group, $P=0.249$; normal control group vs. alcoholic HCC group, $P=0.246$; alcoholic liver cirrhosis group vs. alcoholic HCC group, $P=0.690$ ) (Fig. 5).

\section{Functional biomarker}

Functional biomarker analysis was performed in the alcoholic cirrhosis and alcoholic HCC groups using the Kruskal-

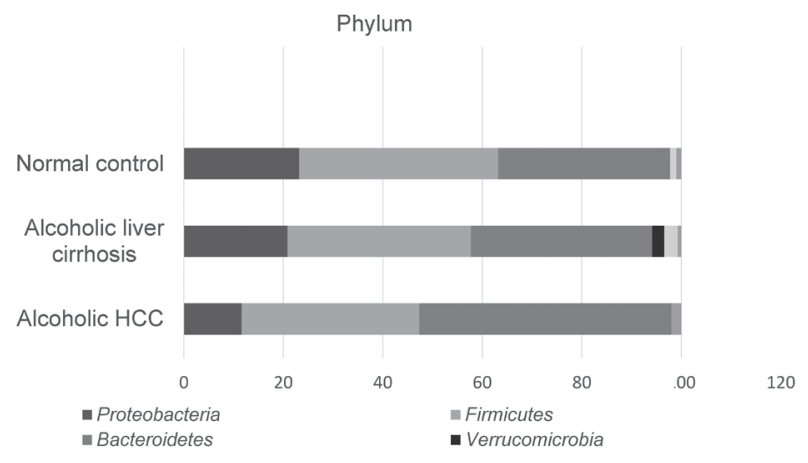

Figure 3. Phylum level averaged taxonomic compositions of the microbial taxonomic profiling sets. HCC, hepatocellular carcinoma.
Wallis $\mathrm{H}$ test. The following six functional biomarkers were significantly different in the alcoholic liver cirrhosis and alcoholic HCC groups: 1) NADH dehydrogenase (obiquinome)

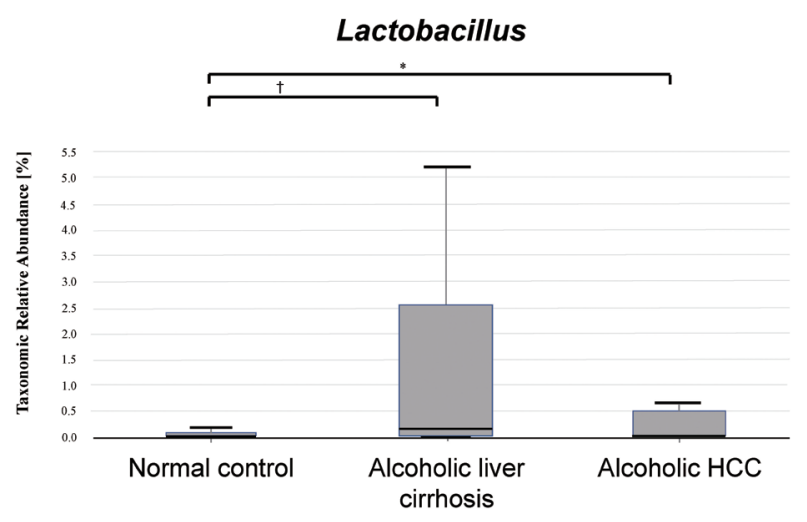

Figure 4. Lactobacillus taxonomic relative abundance in the normal control, alcoholic liver cirrhosis, and alcoholic hepatocellular carcinoma (HCC) groups. Alcoholic liver cirrhosis and alcoholic HCC groups showed increased Lactobacillus compared to that in the normal control group. ${ }^{*} P<0.05 ;{ }^{\dagger} P<0.001$.

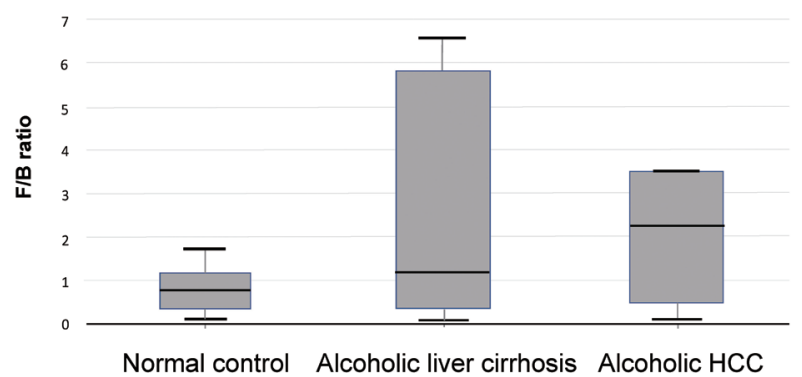

Figure 5. Firmicutes to Bacteroidetes ratio in the normal control, alcoholic liver cirrhosis, and alcoholic hepatocellular carcinoma groups. F/B, Firmicutes/Bacteroidetes.

Table 2. Functional biomarker analysis in alcoholic liver cirrhosis and alcoholic HCC groups

\begin{tabular}{lccc}
\hline Functional biomarker & Alcoholic liver cirrhosis $^{\text {Alcoholic HCC }}$ & $\boldsymbol{P}_{\text {-value }}^{*}$ \\
\hline NADH dehydrogenase Fe-S protein 3 & 0.00021 & 0.00105 & 0.00014 \\
CRP/FNR family transcriptional regulator, global nitrogen regulator & $5.369 \mathrm{e}-6$ & 0.00003 & 0.00020 \\
Vesicle-associated membrane protein 7 & 0.00018 & 0.00042 & 0.00087 \\
Toxin YoeB & 0.01032 & 0.01891 & 0.00094 \\
Peroxisome-assembly ATPase & 0.00010 & 0.00013 & 0.00095 \\
CRP/FNR family transcriptional regulator, nitrogen oxide reductase regulator & $9.982 \mathrm{e}-6$ & 0.00013 & 0.00097 \\
\hline
\end{tabular}

HCC, hepatocellular carcinoma; NADH, nicotinamide adenine dinucleotide-hydrogen; CRP, C-reactive protein; FNR, ferredoxin-NADP+ reductase; NADP, nicotinamide adenine dinucleotide phosphate; ATP, adenosine triphosphate.

"Kruskal-Wallis H test. 
Fe-S protein $3(P=0.00014), 2) \mathrm{CRP} /$ FNR family transcriptional regulator, global nitrogen regulator $(P=0.00020)$,

3) Vesicle-associated membrane protein $7(P=0.00087)$,

4) Toxin YoeB $(P=0.00094), 5)$ Peroxisome-assembly ATPase $(P=0.00095), 6) \mathrm{CRP} / \mathrm{FNR}$ family transcriptional regulator, nitrogen oxide reductase regulator $(P=0.00097)$

(Table 2). We checked AFP and compared it with biomarker. Unfortunately, we did not find any relation between AFP and biomarker.

\section{DISCUSSION}

HCC is a highly complex and heterogeneous disease that affects all populations across the globe. However, the incidence of HCC may vary due to regional and geographical differences in the pervasiveness of causal factors. ${ }^{21}$ HCC has been linked to a multitude of etiological risk factors and cofactors; in approximately $80-90 \%$ of patients, cirrhosis precedes HCC. ${ }^{22,23}$ Of the myriad of factors associated with HCC, the most eminent factors include chronic hepatitis B and $\mathrm{C}$ viral infections, and chronic alcohol consumption. ${ }^{24}$

Another factor that emerged in the past decade is gut dysbiosis. Irrespective of their prominence, disrupted gut barrier function suggests consequences of hepatic cell damage. Moreover, some evidence has shown a link between altered gut microbiota and increased intestinal permeability that can lead to diseased progression at various stages and might promote the progress of HCC throughout all these stages. ${ }^{25}$ The common underlying causes of cirrhosis have been determined as crucial risk factors of HCC. ${ }^{26,27}$ However, HCC can occur in non-cirrhotic livers, which accounts for approximately $20 \%$ of all HCC cases. ${ }^{28}$

In this study, there was a statistically significant difference between the normal control and the alcoholic liver cirrhosis groups $(P<0.001)$ in diversity. Recently, a dramatic relationship was observed between the microbiome and HCC. ${ }^{10} \mathrm{~A}$ previous report suggested that severe alcoholic hepatitis had elevated levels of Actinobacteria and reduced levels of Bacteroidetes. ${ }^{29}$ Our study also revealed reduced levels of Bacteroidetes in the alcoholic cirrhosis group. The microbiome provides a biomarker that can be tested for the risk of a dis- ease and its progression; nevertheless, it remains unknown whether it is the cause or outcome of the disease or whether it is an inferential risk factor or modulator of the disease. Taken together, the microbiome might be related to HCC development and microbiome-related biomarkers are necessary for the diagnosis and treatment of HCC.

In carcinogenesis, cytokines and $\mathrm{T}$-cells are important. The intestinal flora is critically involved in the pathogenesis of HCC by creating an anti-inflammatory microenvironment, which is dependent on liver lipopolysaccharide (LPS). Alistipes, Butyricimonas, Mucispirillum, Oscillibacter, Parabacteroides, Paraprevotella, and Prevotella were classified as enriched genera in this study, among which Oscillibacter species stimulate the differentiation of anti-inflammatory regulatory T-cells (Treg cells) that produce interleukin-10 (IL-10), and Parabacteroides species have proven to withhold inflammation by restraining inflammatory cytokines secretion and promoting the release of anti-inflammatory cytokines IL-10. ${ }^{30,31}$ Along with the aforementioned genera, the species Akkermansia muciniphila, Bacteroides fragilis, Parabacteroides distasonis, and Alistipes shahii were also significantly enriched. Alistipes shahii tends to modulate the gut by abating tumor growth, and Bacteroides fragilis acts by stimulating Treg cells for IL-10 production. ${ }^{32-34}$

Chronic alcohol consumption increases intestinal permeability, leading to high levels of endotoxin, such as LPS, ${ }^{35}$ which is produced by Gram-negative bacteria. LPS is transported directly through the hepatic portal vein, which acts as a pivotal mediator of inflammation in alcoholic liver disease. LPS enables the production of reactive oxygen species and TNF- $\alpha$ activation by Kupffer cells, and leads to inflammation or injury to the liver. In addition, these pro-inflammatory cytokines and LPS cause the release of excess amounts of collagen and $\alpha$-smooth muscle actin, which activates hepatic stellate cells and further promotes fibrosis. ${ }^{36-39}$

Current data from animal and clinical studies point to the gut-liver axis that will bring promising results for primary or secondary prevention of HCC. The microbiome provides biomarkers to test the risk and progression of the disease. Nevertheless, whether it is the cause or effect of this disease or whether it is an estimated risk factor or modulator of the 
disease is unknown. Therefore, such biomarkers have the potential of diagnostic and prognostic mechanisms, a condition that is difficult to achieve. However, in the light of the metagenomic revolution, the study of its composition and function is, after all, an important goal for understanding the progression of cirrhosis to HCC.

In this study, we found that the taxonomic differences between the normal control and alcoholic liver cirrhosis, alcoholic HCC groups. In addition, functional biomarkers with statistically significant differences between the alcoholic liver cirrhosis and the alcoholic HCC groups were identified. Therefore, stool may be a useful diagnostic tool for alcoholic HCC.

\section{AUTHORS' CONTRIBUTION}

Jun Seok: analysis and interpretation of the data, collection and assembly of data, drafting of the article. Ki Tae Suk: conception and design, revision of the article, final approval of the article.

\section{Conflicts of Interest}

The authors declare no conflicts of interest relevant to this article.

\section{REFERENCES}

1. Wong MC, Jiang JY, Goggins WB, Liang M, Fang Y, Fung FD, et al. International incidence and mortality trends of liver cancer: a global profile. Sci Rep 2017;7:45846.

2. Jiang W, Wu N, Wang X, Chi Y, Zhang Y, Qiu X, et al. Dysbiosis gut microbiota associated with inflammation and impaired mucosal immune function in intestine of humans with non-alcoholic fatty liver disease. Sci Rep 2015;5:8096.

3. Cui X, Ye L, Li J, Jin L, Wang W, Li S, et al. Metagenomic and metabolomic analyses unveil dysbiosis of gut microbiota in chronic heart failure patients. Sci Rep 2018:8:635.

4. Kundu P, Blacher E, Elinav E, Pettersson S. Our gut microbiome: the evolving inner self. Cell 2017;171:1481-1493.

5. Lindheim L, Bashir M, Münzker J, Trummer C, Zachhuber V, Leber $B$, et al. Alterations in gut microbiome composition and barrier function are associated with reproductive and metabolic defects in women with polycystic ovary syndrome (PCOS): a pilot study. PLoS
One 2017;12:e0168390.

6. Suk KT, Kim DJ. Gut microbiota: novel therapeutic target for nonalcoholic fatty liver disease. Expert Rev Gastroenterol Hepatol 2019;13:193-204.

7. Hong M, Han DH, Hong J, Kim DJ, Suk KT. Are probiotics effective in targeting alcoholic liver diseases? Probiotics Antimicrob Proteins 2019;11:335-347.

8. Yuan J, Chen C, Cui J, Lu J, Yan C, Wei X, et al. Fatty liver disease caused by high-alcohol-producing klebsiella pneumoniae. Cell Metab 2019;30:675-688. e677.

9. Chen J, Thomsen M, Vitetta L. Interaction of gut microbiota with dysregulation of bile acids in the pathogenesis of nonalcoholic fatty liver disease and potential therapeutic implications of probiotics. J Cell Biochem 2019;120:2713-2720.

10. Petrosino JF, Highlander S, Luna RA, Gibbs RA, Versalovic J. Metagenomic pyrosequencing and microbial identification. Clin Chem 2009;55:856-866.

11. Miele L, Marrone G, Lauritano C, Cefalo C, Gasbarrini A, Day C, et al. Gut-liver axis and microbiota in NAFLD: insight pathophysiology for novel therapeutic target. Curr Pharm Des 2013;19:53145324.

12. Schnabl B, Brenner DA. Interactions between the intestinal microbiome and liver diseases. Gastroenterology 2014;146:1513-1524.

13. Haque TR, Barritt AS 4th. Intestinal microbiota in liver disease. Best Pract Res Clin Gastroenterol 2016;30:133-142.

14. Bajaj JS. The role of microbiota in hepatic encephalopathy. Gut Microbes 2014;5:397-403.

15. Yoon SH, Ha SM, Kwon S, Lim J, Kim Y, Seo H, et al. Introducing EzBioCloud: a taxonomically united database of $16 \mathrm{~S}$ rRNA gene sequences and whole-genome assemblies. Int J Syst Evol Microbiol 2017;67:1613-1617.

16. Edgar RC. Search and clustering orders of magnitude faster than BLAST. Bioinformatics 2010;26:2460-2461.

17. Yoon SH, Ha SM, Kwon S, Lim J, Kim Y, Seo H, et al. Introducing EzBioCloud: a taxonomically united database of $16 \mathrm{~S}$ rRNA gene sequences and whole-genome assemblies. Int J Syst Evol Microbiol 2017;67:1613-1617.

18. Schloss PD, Westcott SL, Ryabin T, Hall JR, Hartmann M, Hollister $E B$, et al. Introducing mothur: open-source, platform-independent, community-supported software for describing and comparing microbial communities. Appl Environ Microbiol 2009;75:7537-7541.

19. Zakrzewski M, Proietti C, Ellis JJ, Hasan S, Brion MJ, Berger B, et al. Calypso: a user-friendly web-server for mining and visualizing microbiome-environment interactions. Bioinformatics 2016;33:782783.

20. Segata N, Izard J, Waldron L, Gevers D, Miropolsky L, Garrett WS, et al. Metagenomic biomarker discovery and explanation. Genome Biol 2011;12:R60. 
21. Farazi PA, DePinho RA. Hepatocellular carcinoma pathogenesis: from genes to environment. Nat Rev Cancer 2006;6:674-687.

22. Ghouri YA, Mian I, Rowe JH. Review of hepatocellular carcinoma: epidemiology, etiology, and carcinogenesis. J Carcinog 2017;16:1.

23. Zhang DY, Friedman SL. Fibrosis-dependent mechanisms of hepatocarcinogenesis. Hepatology 2012;56:769-775.

24. Shlomai A, de Jong YP, Rice CM. Virus associated malignancies: the role of viral hepatitis in hepatocellular carcinoma. Semin Cancer Biol 2014;26:78-88.

25. Yu LX, Schwabe RF. The gut microbiome and liver cancer: mechanisms and clinical translation. Nat Rev Gastroenterol Hepatol 2017;14:527-539.

26. Chitapanarux T, Phornphutkul K. Risk factors for the development of hepatocellular carcinoma in Thailand. J Clin Transl Hepatol 2015;3:182-188.

27. Llovet JM. Updated treatment approach to hepatocellular carcinoma. J Gastroenterol 2005;40:225-235.

28. Alkofer B, Lepennec V, Chiche L. Hepatocellular cancer in the noncirrhotic liver. J Visc Surg 2011;148:3-11.

29. Ciocan D, Voican CS, Wrzosek L, Hugot C, Rainteau D, Humbert $\mathrm{L}$, et al. Bile acid homeostasis and intestinal dysbiosis in alcoholic hepatitis. Aliment Pharmacol Ther 2018;48:961-974.

30. Arpaia N, Campbell C, Fan X, Dikiy S, van der Veeken J, deRoos P, et al. Metabolites produced by commensal bacteria promote peripheral regulatory T-cell generation. Nature 2013;504:451-455.

31. Kverka M, Zakostelska Z, Klimesova K, Sokol D, Hudcovic T, Hrncir $T$, et al. Oral administration of parabacteroides distasonis antigens attenuates experimental murine colitis through modulation of immunity and microbiota composition. Clin Exp Immunol
2011;163:250-259.

32. Round JL, Mazmanian SK. Inducible Foxp3+ regulatory T-cell development by a commensal bacterium of the intestinal microbiota. Proc Natl Acad Sci U S A 2010;107:12204-12209.

33. lida N, Dzutsev A, Stewart CA, Smith L, Bouladoux N, Weingarten RA, et al. Commensal bacteria control cancer response to therapy by modulating the tumor microenvironment. Science 2013;342:967-970.

34. Li J, Sung CY, Lee N, Ni Y, Pihlajamäki J, Panagiotou G, et al. Probiotics modulated gut microbiota suppresses hepatocellular carcinoma growth in mice. Proc Natl Acad Sci U S A 2016;113:E1306E1315.

35. Rao RK, Seth A, Sheth P. Recent Advances in Alcoholic Liver Disease I. Role of intestinal permeability and endotoxemia in alcoholic liver disease. Am J Physiol Gastrointest Liver Physiol 2004;286:G881-G884.

36. Suraweera DB, Weeratunga AN, Hu RW, Pandol SJ, Hu R. Alcoholic hepatitis: The pivotal role of Kupffer cells. World J Gastrointest Pathophysiol 2015;6:90-98.

37. Trebicka J, Krag A, Gansweid S, Appenrodt B, Schiedermaier P, Sauerbruch $T$, et al. Endotoxin and tumor necrosis factor-receptor levels in portal and hepatic vein of patients with alcoholic liver cirrhosis receiving elective transjugular intrahepatic portosystemic shunt. Eur J Gastroenterol Hepatol 2011;23:1218-1225.

38. Wheeler MD. Endotoxin and Kupffer cell activation in alcoholic liver disease. Alcohol Res Health 2003;27:300-306.

39. Gao B. Hepatoprotective and anti-inflammatory cytokines in alcoholic liver disease. J Gastroenterol Hepatol 2012;27 Suppl 2:89-93. 